\title{
Investigation of effective system designs for transcranial photoacoustic tomography of the brain
}

Kenji Mitsuhashi, Robert W. Schoonover, Chao Huang, Lihong V. Wang, Mark A. Anastasio

Kenji Mitsuhashi, Robert W. Schoonover, Chao Huang, Lihong V. Wang, Mark A. Anastasio, "Investigation of effective system designs for transcranial photoacoustic tomography of the brain," Proc. SPIE 8943, Photons Plus Ultrasound: Imaging and Sensing 2014, 894369 (3 March 2014); doi: $10.1117 / 12.2041998$ 


\title{
Investigation of effective system designs for transcranial photoacoustic tomography of the brain
}

\author{
Kenji Mitsuhashi $^{a}$, Robert W. Schoonover ${ }^{a}$, Chao Huang ${ }^{a}$, Lihong V. Wang ${ }^{a}$, \\ and Mark A. Anastasio ${ }^{*}$ \\ ${ }^{a}$ Department of Biomedical Engineering, Washington University in St. Louis, St. Louis, MO \\ 63130, USA
}

\begin{abstract}
Photoacoustic computed tomography (PACT) holds great promise for transcranial brain imaging. However, the strong reflection, scattering and attenuation of acoustic waves in the skull present significant challenges to developing this method. We report on a systematic computer-simulation study of transcranial brain imaging using PACT. The goal of this study was to identify an effective imaging system design that can be translated for clinical use. The propagation of photoacoustic waves through a model skull was studied by use of an elastic finite-difference time-domain (FDTD) method. The acoustic radiation pattern from a photoacoustic source just beneath the skull was observed with a ring transducer array that was level with the source. The observed radiation pattern was found to contain stronger contributions from waves that were converted to shear waves in skull than longitudinal waves that did not undergo mode conversion. Images reconstructed from the pressure data that contain shear wave components possess better resolution than images reconstructed from the data that only contain the longitudinal wave signals. These observations revealed that the detection system should be designed to capture photoacoustic signals that travel through the skull in the form of shear waves as well as in the form of longitudinal waves. A preliminary investigation on the effect of the presence of absorption in the skull is also reported. This study provides an insight into the wave phenomena in transcranial PACT imaging, as well as a concrete detection design strategy that mitigates the degraded resolution of reconstructed images.
\end{abstract}

Keywords: Photoacoustic computed tomography, Image reconstruction, Brain imaging

\section{INTRODUCTION}

Photoacoustic computed tomography (PACT), also known as optoacoustic tomography, is a non-invasive, hybrid computed imaging modality that combines the rich contrast of optical imaging methods with the deep penetration and high spatial resolution of ultrasound imaging methods. ${ }^{1-3}$ In PACT, the target is illuminated with a short laser pulse, and elastic waves are generated in the surrounding medium via the photoacoustic effect. Ultrasonic transducers are subsequently employed to measure the photoacoustic signals at a number of locations outside the object. From these data, a PACT image reconstruction algorithm is employed to produce an image that depicts the spatially variant absorbed optical energy density within the object.

A successful use of PACT has been demonstrated in functional imaging of mouse brains. ${ }^{4}$ For animals with thicker skulls, PACT provides images that qualitatively depict large vascular structures of the brain. ${ }^{5}$ However, the propagation of elastic waves through the skull induces significant changes to the photoacoustic signals because of the presence of the strong refraction, reflection and attenuation of elastic waves in cortical bone. These effects can result in reconstructed images with distortions and degraded spatial resolution. In recent studies, algorithms that compensate for the heterogeneity of the speed of sound (SOS) have been developed for reconstructing PACT images, ${ }^{6,7}$ which may benefit transcranial PACT applications.

In this article, we report on a systematic computer-simulation study of transcranial brain imaging using 3D PACT that demonstrates the feasibility of an imaging system that utilizes shear waves as well as longitudinal waves. The imaging system is shown to alleviate the distortions and degraded resolution of reconstructed images

Further additional information contact:

K.M.: kenji.mitsuhashi@wustl.edu M.A.A.: anastasio@wustl.edu

Photons Plus Ultrasound: Imaging and Sensing 2014, edited by Alexander A. Oraevsky, Lihong V. Wang, Proc. of SPIE Vol. 8943, 894369 - (C) 2014 SPIE · CCC code: 1605-7422/14/\$18 - doi: 10.1117/12.2041998 
without computational compensation for the SOS heterogeneity. The propagation of photoacoustic waves through a model skull is studied by use of an elastic finite-difference time-domain (FDTD) method. The acoustic radiation pattern from a photoacoustic source just beneath the skull is observed with a ring transducer array that is level with the source. The observed radiation pattern is found to contain more of the waves that come through the skull in the form of shear waves than the waves that come in the form of longitudinal waves. Images reconstructed from the pressure data that contain shear wave components possess better resolution than images reconstructed from the data that only contain the longitudinal wave signals. These observations reveal that the detection system can be designed to capture photoacoustic signals that travel through the skull in the form of shear waves as well as in the form of longitudinal waves. A preliminary investigation on the effect of the presence of absorption in the skull is also reported. This study provides an insight into the wave phenomena in transcranial PACT imaging, as well as a concrete detection design strategy that mitigates the degraded resolution of reconstructed images.

\section{BACKGROUND}

Below we review the salient features of the imaging physics, the method of elastic FDTD and convolutional perfectly-matched layer (CPML), and the image reconstruction algorithm that will be employed in our studies. ${ }^{8-12}$

\subsection{Imaging physics}

In PACT, a pulsed laser is employed to irradiate the object, and the photoacoustic effect results in the generation of a stress tensor field $\sigma(\mathbf{r}, t)$, where $\mathbf{r} \in \mathbb{R}^{3}$ and $t$ are spatial and temporal coordinates, respectively. In this work, the to-be-imaged object and the surrounding medium are assumed to possess a linear isotropic elasticity described by Hooke's law. Additionally, an empirical diffusive absorption model is employed for modeling the absorption of elastic waves in the cortical bone. ${ }^{13}$ Under these conditions, the photoacoustic field $\sigma(\mathbf{r}, t)$ and the corresponding velocity vector field $\mathbf{u}(\mathbf{r}, t)$ satisfy the first-order elastic wave equations ${ }^{11}$

$$
\begin{aligned}
& \frac{\partial \mathbf{u}(\mathbf{r}, t)}{\partial t}=-\alpha(\mathbf{r}) \mathbf{u}(\mathbf{r}, t)+\frac{1}{\rho(\mathbf{r})} \nabla \cdot \sigma(\mathbf{r}, t), \\
& \frac{\partial \sigma(\mathbf{r}, t)}{\partial t}=\lambda(\mathbf{r})(\nabla \cdot \mathbf{u}(\mathbf{r}, t)) I+2 \mu(\mathbf{r}) D(\mathbf{r}, t),
\end{aligned}
$$

subject to the initial conditions

$$
\begin{aligned}
& \left.\mathbf{u}(\mathbf{r}, t)\right|_{t=0}=0,\left.\quad \frac{\partial \mathbf{u}(\mathbf{r}, t)}{\partial t}\right|_{t=0}=0, \\
& \left.\sigma(\mathbf{r}, t)\right|_{t=0}=-\Gamma A(\mathbf{r}) I,\left.\quad \frac{\partial \sigma(\mathbf{u}, t)}{\partial t}\right|_{t=0}=0,
\end{aligned}
$$

where $\nabla$ denotes the spatial differential operator, $\alpha(\mathbf{r})$ is the absorption coefficient, $\rho(\mathbf{r})$ is the density, $\lambda(\mathbf{r})$ and $\mu(\mathbf{r})$ are the Lamé parameters, $I$ is the identity tensor, $D(\mathbf{r}, t)$ is the strain rate tensor defined as

$$
D(\mathbf{r}, t)=\frac{1}{2}\left(\nabla \mathbf{u}(\mathbf{r}, t)+\nabla \mathbf{u}(\mathbf{r}, t)^{\mathrm{T}}\right),
$$

where $\nabla \mathbf{u}(\mathbf{r}, t)^{\mathrm{T}}$ denotes the transpose of the tensor $\nabla \mathbf{u}(\mathbf{r}, t), A(\mathbf{r})$ is a compactly supported and bounded function that represents the absorbed optical energy density, and $\Gamma$ is the Grüneisen coefficient, which represents the photoacoustic effect. ${ }^{1}$ Here, we assume that the Grüneisen coefficient is constant because $A(\mathbf{r})$ is assumed to be supported within soft tissue in this work, and thus the variation of the coefficient is negligible. The photoacoustic field is subsequently measured with ultrasonic transducer elements placed outside the object. Since we assume that all transducer elements are located in the medium with $\mu=0$ (no support for shear wave propagation; additional details described in Section 3.1), the photoacoustic field in the medium in which the transducer elements reside is identified as an isotropic pressure field $\sigma(\mathbf{r}, t)=-p(\mathbf{r}, t) I=\frac{1}{3} \operatorname{tr} \sigma(\mathbf{r}, t) I$. Thus, the pressure signals measured at the transducer locations are given by

$$
p(\mathbf{r}, t)=-\frac{1}{3} \operatorname{tr} \sigma(\mathbf{r}, t) .
$$

In a mathematical sense, the goal of PACT is to determine $A(\mathbf{r})$ from knowledge of $p(\mathbf{r}, t)$. 


\subsection{Method of elastic FDTD and CPML}

The first-order elastic wave equations (1) and (2) are discretized by use of a staggered grid. ${ }^{9-11}$ The temporal derivatives that appear on the left-hand side of the equations are approximated as second-order temporal finitedifferences (FDs), and the spatial derivatives on the right-hand side as fourth-order spatial FDs. The discretized velocity vector field is defined at the time points $t=0, \Delta t, 2 \Delta t, \ldots$ while the stress tensor field is defined at the time points $t=\frac{1}{2} \Delta t, \frac{3}{2} \Delta t, \frac{5}{2} \Delta t, \ldots$, where $\Delta t$ denotes the time step. Therefore, in each time step, the velocity vector field is updated first, and then the stress tensor field is updated based on knowledge of the updated velocity vector field. The reader is referred to Ref. 11 for a thorough review of the classical elastic FDTD method.

The stress tensor field and the velocity vector field are assumed to be defined in an infinite medium in this work. To simulate the fields by use of a finite-size computational volume, we employ the unsplit CPML technique ${ }^{12}$ to avoid artificial reflection and/or distortion of photoacoustic waves at the boundary of the computational volume. The technique introduces absorbing boundary layers that each modify the spatial derivatives along the direction perpendicular to the layer as

$$
\partial_{\xi}=\frac{1}{\kappa_{\xi}} \partial_{\xi}+\psi_{\xi}
$$

where the subscript $\xi$ denotes the direction, $\kappa_{\xi} \geq 1$ is a damping coefficient, and $\psi_{\xi}$ is a memory variable that satisfies the time-evolution equation

$$
\psi_{\xi}^{(n+1)}=b_{\xi} \psi_{\xi}^{(n)}+a_{\xi}\left(\partial_{\xi}\right)^{\left(n+\frac{1}{2}\right)},
$$

where the superscripts, e.g. $(n)$, denote the time step, and $a_{\xi}$ and $b_{\xi}$ are defined as

$$
a_{\xi}=\frac{d_{\xi}}{\kappa_{\xi}\left(d_{\xi}+\kappa_{\xi} \alpha_{\xi}\right)}\left(b_{\xi}-1\right), \quad b_{\xi}=\mathrm{e}^{-\left(\frac{d_{\xi}}{\kappa_{\xi}}+\alpha_{\xi}\right) \Delta t} .
$$

Here, $d_{\xi} \geq 0$ and $\alpha_{\xi} \geq 0$ are damping coefficients. We refer the reader to Ref. 12 for the additional details of the CPML technique, e.g. the optimal values of the damping coefficients.

\subsection{Image reconstruction}

In this work, a widely-employed back-projection algorithm is used for reconstructing PACT images. ${ }^{8,14}$ The absorbed optical energy density $A(\mathbf{r})$ is reconstructed from the pressure data measured at $N$ transducer locations that are each specified by $\mathbf{r}_{n}(n=1, \ldots, N)$ as

$$
A^{\mathrm{r}}(\mathbf{r})=\left.\frac{1}{\Gamma} \sum_{n=1}^{N} \frac{\Delta \Omega_{n}}{\Omega_{0}}\left(2 p\left(\mathbf{r}_{n}, t\right)-2 t \frac{\partial p\left(\mathbf{r}_{n}, t\right)}{\partial t}\right)\right|_{t=\left\|\mathbf{r}-\mathbf{r}_{n}\right\| / c_{\text {eff }}},
$$

where the superscript ' $\mathrm{r}$ ' denotes that $A^{\mathrm{r}}(\mathbf{r})$ is a reconstruction of $A(\mathbf{r}), \Delta \Omega_{n}$ is the solid angle of the $n$th transducer defined as $\Delta \Omega_{n}=\mathbf{n}_{n} \cdot\left(\mathbf{r}-\mathbf{r}_{n}\right) /\left\|\mathbf{r}-\mathbf{r}_{n}\right\|^{3}$, where $\mathbf{n}_{n}$ is the normal vector of the $n$th transducer, $\Omega_{0}$ is the total solid angle defined as $\Omega_{0}=\sum_{n=1}^{N} \Delta \Omega_{n}$, and $c_{\text {eff }}$ is the effective SOS. For use of comparison, $A(\mathbf{r})$ in the horizontal ( $x y$ ) plane that passes through the center of the object is reconstructed.

\section{COMPUTER-SIMULATION STUDIES}

Computer simulation studies were conducted to systematically investigate the feasibility of an imaging system that utilizes shear waves as well as longitudinal waves.

\subsection{Imaging system configuration}

To demonstrate the improvement of resolution owing to the presence of shear waves, we considered a 3D imaging configuration that was based on a spherical model skull (Fig. 1). The model skull was a truncated spherical shell with an outer diameter of $200 \mathrm{~mm}$ and a thickness of $5 \mathrm{~mm}$ that was placed within a rectangular computational volume with a size of $278.4 \mathrm{~mm}(x) \times 278.4 \mathrm{~mm}(y) \times 91.2 \mathrm{~mm}(z)$. The computational volume consisted of $928(x)$ $\times 928(y) \times 304(z)$ cells with a $0.3 \mathrm{~mm}$ interval that each retain the value of $\sigma(\mathbf{r}, t), \mathbf{u}(\mathbf{r}, t)$, and other material 
property variables. The volume was enclosed with 16 layers of CPML. 100 point-like transducer elements were employed to form a ring transducer array with a radius of $110 \mathrm{~mm}$. Each transducer element was assumed to have a normal vector pointing to the center of the ring. The to-be-imaged object was a 3D Gaussian blob defined as

$$
A(\mathbf{r})=\mathrm{e}^{-\frac{\left\|\mathbf{r}-\mathbf{r}_{0}\right\|^{2}}{2 s^{2}}},
$$

where $s=1.5 \mathrm{~mm}$ denotes the standard deviation and $\mathbf{r}_{0}$ denotes the center of the blob that was located 15 $\mathrm{mm}$ beneath the top of the skull, i.e. $10 \mathrm{~mm}$ beneath the inner surface of the skull. The ring transducer array and the object were concentric. The skull was assumed to be made of solid cortical bone that supports shear wave propagation and placed within a water-like medium that does not support shear wave propagation. The elasto-acoustic properties of cortical bone and the water-like medium are summarized in Table 1. Notice that the SOS of shear waves in cortical bone is equal to the SOS of longitudinal waves in the water-like medium. ${ }^{13}$ The Grüneisen parameter $\Gamma$ in the object was assumed to have a value of 2000.

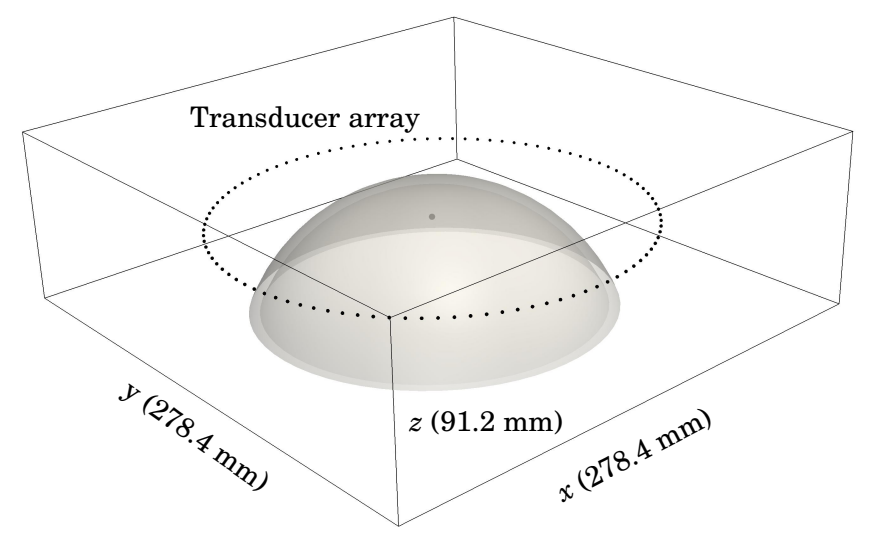

Figure 1. The 3D imaging system configuration based on a spherical model skull. The model skull is depicted as a semi-transparent, gray truncated spherical shell placed within a rectangular computational volume with a size of 278.4 $\mathrm{mm}(x) \times 278.4 \mathrm{~mm}(y) \times 91.2 \mathrm{~mm}(z)$. The ring transducer array consists of 100 point-like transducer elements.

\begin{tabular}{lcc}
\hline \hline Material parameter & Cortical bone & Water-like medium \\
\hline Density $(\rho)$ & $1850 \mathrm{~kg} / \mathrm{m}^{3}$ & $1000 \mathrm{~kg} / \mathrm{m}^{3}$ \\
SOS of longitudinal waves $\left(c_{l}\right)$ & $3000 \mathrm{~m} / \mathrm{s}$ & $1500 \mathrm{~m} / \mathrm{s}$ \\
SOS of shear waves $\left(c_{s}\right)$ & $1500 \mathrm{~m} / \mathrm{s}$ & - \\
\hline \hline
\end{tabular}

Table 1. The elasto-acoustic properties of cortical bone and the water-like medium ${ }^{13}$

\subsection{Numerical details}

Due to the large size of the computational volume $(\approx 260$ million cells $)$, FDTD simulations of the propagation of photoacoustic waves are computationally burdensome and demanding in terms of the amount of memory. Therefore, we implemented an elastic FDTD algorithm as a GPU-based massively parallel algorithm whose basic design was inspired by Refs. 10 and 15 and briefly described as follows.

Let $G$ be the number of GPU units. The computational volume $\boldsymbol{\theta}$ is divided by $x z$-planes into $G$ equal-sized sub-volumes $\left\{\boldsymbol{\theta}^{g}\right\}_{g=1}^{G}$ that are specified by the index $g=1, \ldots, G$. Each sub-volume $\boldsymbol{\theta}^{g}$ is given an additional 2 layers of cells at each interface with the adjacent sub-volume, which we refer to as margin layers. The subvolumes $\left\{\boldsymbol{\theta}^{g}\right\}_{g=1}^{G}$ are distributed to $G$ CPU threads that each manage one GPU unit. In each thread, $\boldsymbol{\theta}^{g}$ is loaded into the global memory of the GPU unit. To minimize the number of accesses to the global memory, the sub-volume is further divided into a collection of two-dimensional (2D) $x z$-tiles that each have $16 \times 16$ cells, 
and the tiles are consecutively loaded into the shared memory. The values of the tile loaded into the shared memory are parallelly updated by use of $256(=16 \times 16)$ GPU threads and sent back to the global memory on the completion of the update. Each time step is executed in two phases. In the first phase, the GPU computes the values of the tiles that correspond to the margin layers of the adjacent sub-volume. The result is copied into the margin layers of the adjacent sub-volume via the message passing interface (MPI). In the second phase, the GPU unit computes the values of the remaining tiles using the values of the margin layers that come from the adjacent sub-volume. In either phase, the velocity vector field is updated first based on the discretized version of the Eq. (1), and then the stress tensor field is updated based on Eq. (2).

The GPU-based FDTD routine and the CPU-based MPI routine were implemented in CUDA (NVIDIA, USA) and C++ with the Boost.MPI library, respectively. All simulations were executed on a GPU cluster with 8 GPU units (Tesla C1060; NVIDIA, USA).

The time step $\Delta t$ was chosen so that the Courant-Friedrichs-Lewy (CFL) number $C=c_{\max } \Delta t / \Delta x$ had a value of 0.1 , where $c_{\max }$ is the maximum SOS in the system, and $\Delta x$ denotes the spatial interval of the cell. This means that $\Delta t=10 \mathrm{~ns}$. The total number of time steps computed in each simulation was 15000 .

\section{RESULTS}

\subsection{Cone region}

The propagation of photoacoustic waves is illustrated in Fig. 2. For comparison purposes, the propagation of photoacoustic waves through a skull that does not support shear wave propagation is also depicted. The snapshots for $t=23 \mu \mathrm{s}$ show that the radiation pattern of the waves that travel through the skull in the form of longitudinal waves, which we refer to the L-waves, was highly asymmetric. The L-waves propagating towards the upward direction possessed large amplitudes while the L-waves propagating towards lateral directions possessed small amplitudes. This difference is explained by the occurrence of total internal reflection at the interface of cortical bone and the water-like medium. Since the SOS of longitudinal waves in the water-like medium $\left(c_{w, l}=1500 \mathrm{~m} / \mathrm{s}\right)$ is much smaller than that in cortical bone $\left(c_{b, l}=3000 \mathrm{~m} / \mathrm{s}\right)$, longitudinal waves that enter the skull with a large incident angle $\left(\gtrsim \arcsin c_{w, l} / c_{b, l}=30\right.$ deg.) are totally reflected and thus remain inside the skull. As a consequence, the L-waves that travel through the skull mainly propagate within a cone-shaped region pointing upwards. We will refer to this cone-shaped region as the "cone region."

An observation of the snapshots for $t=60 \mu$ s indicates that the presence of shear waves in the skull further reduced the amplitude of the L-waves that reached the transducer array. The waves that travel through the skull in the form of shear waves, which we refer to as S-waves, were more prominent when a skull with shear-wave support was employed. Notice that the S-waves possess a sharper wavefront than the L-waves. This is due to the fact that the L-waves travel through a limited aperture that is formed by the occurrence of total internal reflection. By contrast, the S-waves do not experience such a limited aperture because the SOS of longitudinal waves in the water-like medium $\left(c_{w, l}=1500 \mathrm{~m} / \mathrm{s}\right)$ is equal to the SOS of shear waves in cortical bone $\left(c_{b, s}=1500\right.$ $\mathrm{m} / \mathrm{s})$. This observation will explain the better resolution achieved by the imaging system that utilizes S-waves as well as L-waves.

\subsection{Presence of shear waves improves the accuracy of reconstructed images}

Since the propagation of L-waves is spatially restricted within a cone region due to the occurrence of total internal reflection, PACT imaging systems that are designed with only L-waves considered in the model inevitably suffer from degraded resolution because of the limited aperture effect. To overcome this difficulty, we propose the use of imaging systems that are designed with the mode conversion to S-waves in the skull in mind and reconstruct images from the information that the S-waves convey through the skull. Fig. 1 shows an example of such imaging systems, in which all transducer elements are placed outside the cone region so that they mainly capture waves that were mode-converted to S-waves in the skull.

To demonstrate that designing a system with the mode conversion to S-waves modeled improves the accuracy of reconstructed images, images reconstructed from simulated photoacoustic signals that travel through skulls with different shear moduli were compared (Fig. 3). The reconstructed images are specified by the SOS of shear waves in cortical bone $\left(c_{b, s}\right)$, which is given by $c_{b, s}=\sqrt{\mu / \rho}$, where $\mu$ is the shear modulus of cortical bone. 


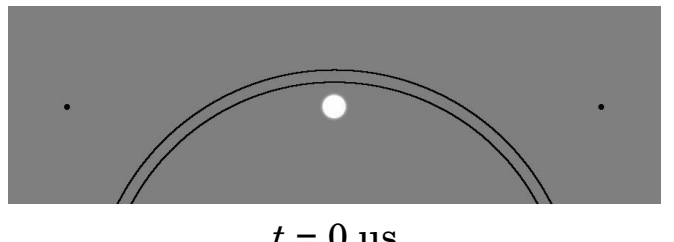

Average stress (= - $\operatorname{tr} \sigma / 3)$

$-10 \square 10$

$$
t=0 \mu \mathrm{s}
$$

Shear wave propagation supported

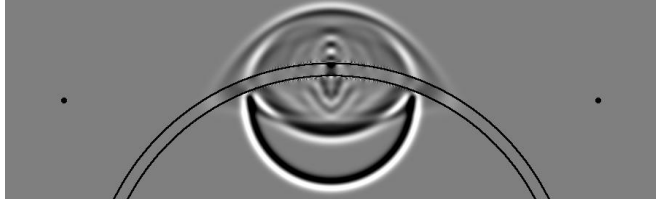

$t=23 \mu \mathrm{s}$

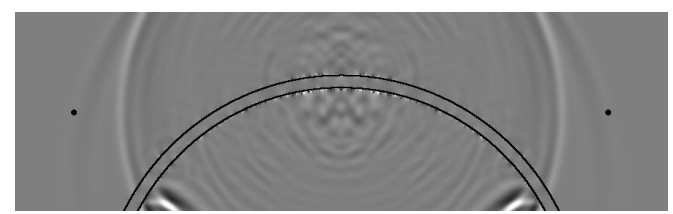

$t=60 \mu \mathrm{s}$

Shear wave propagation NOT supported

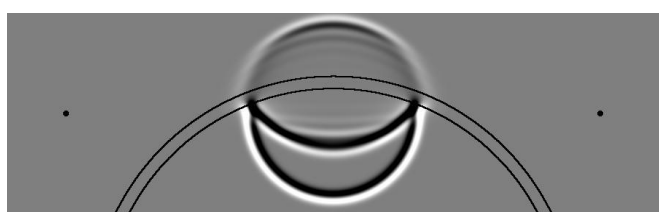

$t=23 \mu \mathrm{s}$

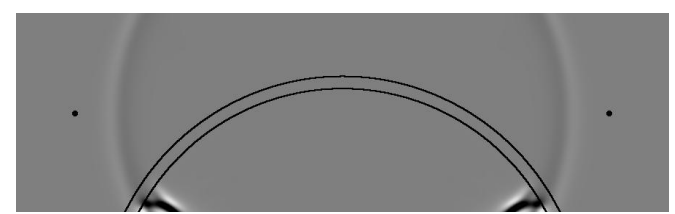

$t=60 \mu \mathrm{s}$

Figure 2. The average stress, defined as $-\operatorname{tr} \sigma / 3$, which is identical to the pressure when evaluated in the water-like medium, is displayed for demonstrating the propagation of photoacoustic waves through the skull. The spherical shell (shown in black) represents the cross-section of the skull in the $x z$-plane that passes through the center of the object. The black dots on both sides of the skull show the location of the ring transducer array.

For each reconstructed image, the effective SOS $c_{\text {eff }}$ in the back-projection formula (10) was determined so that it minimized the root-mean-square error (RMSE) between the reconstructed image and the true object. As expected, the image reconstructed from the signals for a larger shear modulus was shown to possess a better resolution than the image reconstructed from the signals for a smaller shear modulus. This observation indicates that incorporating S-waves into the imaging model improves the accuracy of reconstructed images.

\subsection{Accuracy of reconstructed images in the presence of absorption}

Finally, we present the preliminary results that demonstrate the accuracy of reconstructed images in the presence of absorption in the skull (Fig. 4). Images reconstructed from simulated photoacoustic signals that travel through skulls with different absorption coefficients $(\alpha)$ were compared. In this study, the SOS of shear waves in the skull was fixed at $1500 \mathrm{~m} / \mathrm{s}$. The value of $\alpha$ for actual cortical bone has been reported as $\alpha=0.96 / \mu \mathrm{s} .{ }^{13}$ While the image reconstructed from the signals for $\alpha=0.5 / \mu$ s exhibited no significant change in resolution, the image for $\alpha=1.0 / \mu$ s possessed a slightly degraded resolution compared to the image for $\alpha=0.0 / \mu$ s. However, the resolution of the image for $\alpha=1.0 / \mu \mathrm{s}$ was still better than the resolution of the image that was reconstructed from the L-wave signals (cf. Fig. 3). These results show that imaging systems designed with an elastic wave propagation model produce more accurate reconstructed images than imaging systems based on L-waves only, even in the presence of absorption in the skull.

\section{CONCLUSIONS}

PACT imaging holds a great promise in transcranial brain imaging owing to its rich optical contrast and deep nonionizing penetration. ${ }^{4,5}$ However, the propagation of photoacoustic waves through the skull introduces significant changes to the observed signals because of the presence of the strong refraction, reflection and attenuation of photoacoustic waves in the skull. These changes can result in distortions and degraded resolution of reconstructed 


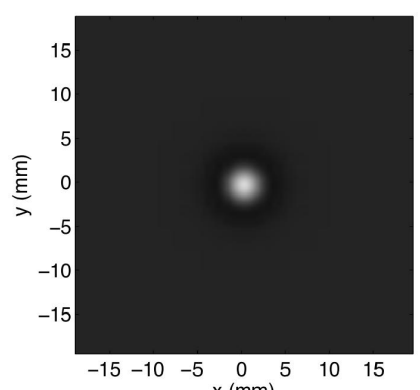

No Skull
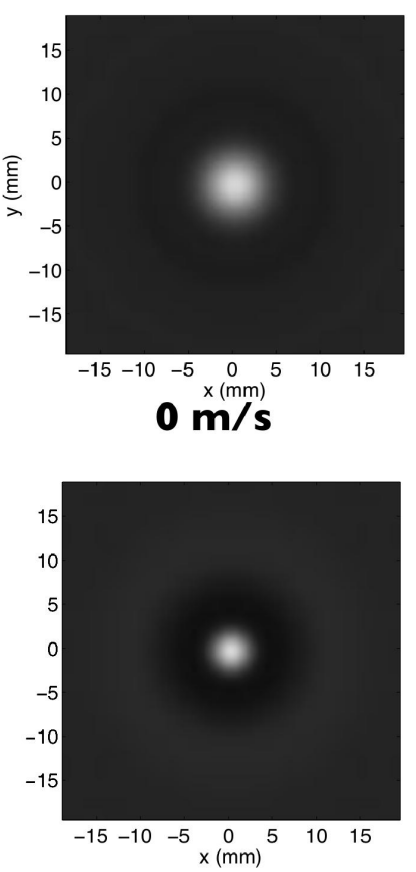

$1000 \mathrm{~m} / \mathrm{s}$
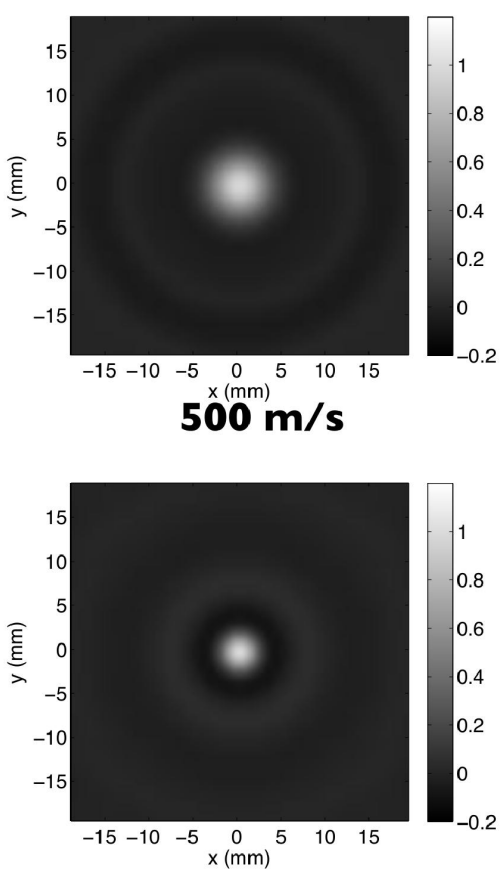

$1500 \mathrm{~m} / \mathrm{s}$

Figure 3. Images reconstructed from simulated photoacoustic signals that travel through skulls with different shear moduli. The images are specified by the SOS of shear waves in cortical bone $\left(c_{b, s}\right)$, which is given by $c_{b, s}=\sqrt{\mu / \rho}$, where $\mu$ is the shear modulus. The image reconstructed from the signals for a larger shear modulus possessed a better resolution than the image reconstructed from the signals for a smaller shear modulus.
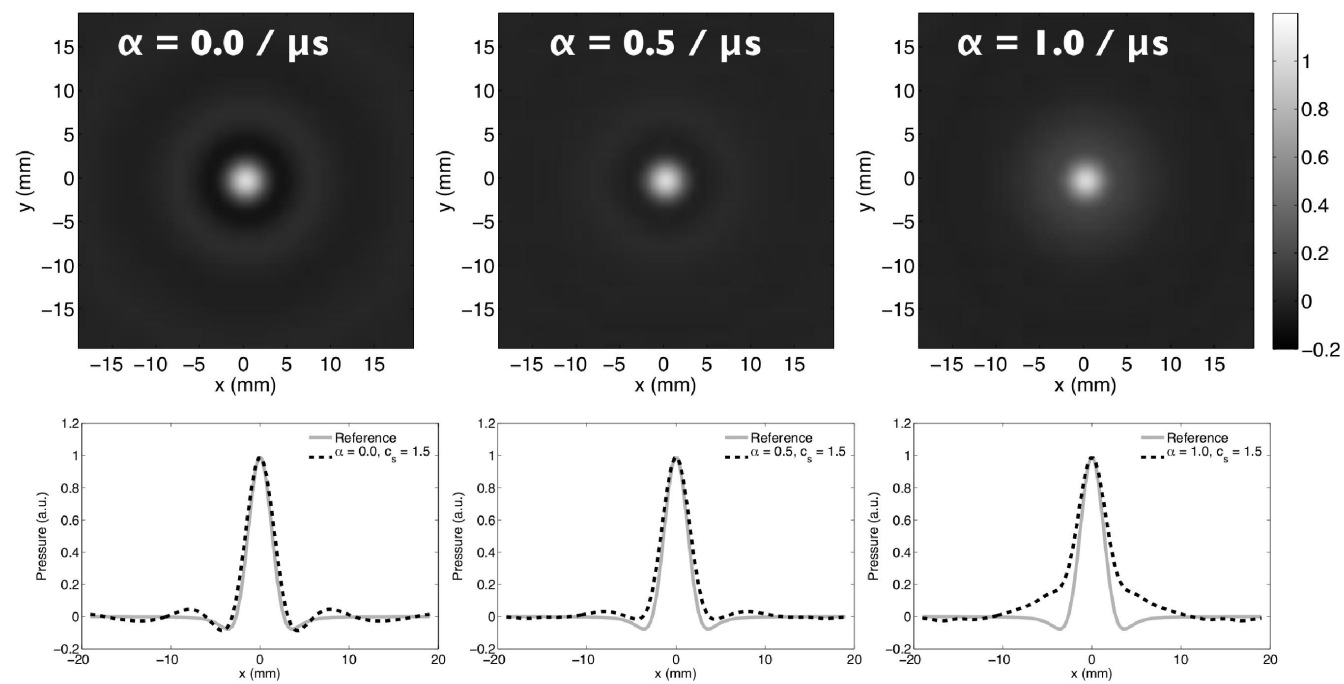

Figure 4. Images reconstructed from simulated photoacoustic signals that travel through skulls with different absorption coefficients. The SOS of shear waves in cortical bone was fixed at $1500 \mathrm{~m} / \mathrm{s}$. The image reconstructed from the signals for $\alpha=1.0 / \mu$ s possessed a slightly degraded resolution, but the resolution was still better than the resolution of the image reconstructed from L-wave signals (Fig. 3). 
images. To mitigate the degraded resolution of reconstructed images, reconstruction algorithms that compensate for the SOS heterogeneity in the skull have been reported, ${ }^{6,16}$ but they can be computationally burdensome for clinical use. The goal of this study was to demonstrate the effectiveness of the use of an imaging system that incorporates the mode conversion to S-waves in the skull into the imaging model for compensating for the degraraded resolution of reconstructed images without burdensome computation.

The computer-simulation studies described above show that the use of an imaging system with the mode conversion to S-waves in the skull modeled successfully mitigated the degraded resolution of reconstructed images introduced by the presence of the skull. The propagation of L-waves was found to be spatially restricted within a cone region, and thus imaging systems that are designed with only L-waves considered in the imaging model inevitably suffer from degraded resolution because of the limited aperture effect. This observation indicates that imaging systems that employ transducer elements located outside the cone region mainly capture waves that were mode-converted to S-waves in the skull and can reconstruct images from the information that the S-waves convey through the skull. We demonstrated that images reconstructed by use of an imaging system with the mode conversion to S-waves considered in the imaging model possessed a better resolution than images reconstructed by use of an imaging system that is designed with only L-waves considered. The preferable nature of the S-wavebased imaging system remained valid in the presence of absorption in the skull. Since the S-wave-based imaging system employs a back-projection algorithm for reconstructing images, it is computationally very efficient and readily applicable for clinical use. This study provides an insight into the wave phenomena in transcranial PACT imaging, as well as a concrete detection design strategy that mitigates the degraded resolution of reconstructed images.

\section{ACKNOWLEDGMENTS}

This work was supported in part by NIH awards EB010049 and EB014617 (F32).

\section{REFERENCES}

[1] Xu, M. and Wang, L. V., "Photoacoustic imaging in biomedicine," Rev. Sci. Instrum. 77(4), 041101 (2006).

[2] Oraevsky, A. A. and Karabutov, A. A., "Optoacoustic tomography," in [Biomedical photonics handbook], Vo-Dinh, T., ed., 34/1-34/34, CRC Press, Boca Raton (2003).

[3] Wang, K. and Anastasio, M. A., "Photoacoustic and thermoacoustic tomography: image formation principles," in [Handbook of mathematical methods in imaging], Scherzer, O., ed., 781-815, Springer, New York (2011).

[4] Wang, X., Pang, Y., Ku, G., Xie, X., Stoica, G., and Wang, L. V., "Noninvasive laser-induced photoacoustic tomography for structural and functional in vivo imaging of the brain," Nat. Biotechnol. 21(7), 803-806 (2003).

[5] Xu, Y. and Wang, L. V., "Rhesus monkey brain imaging through intact skull with thermoacoustic tomography," IEEE T. Ultrason. Ferr. 53(3), 542-548 (2006).

[6] Treeby, B. E., Zhang, E. Z., and Cox, B. T., "Photoacoustic tomography in absorbing acoustic media using time reversal," Inverse Probl. 26(11), 115003 (2010).

[7] Huang, C., Wang, K., Nie, L., Wang, L. V., and Anastasio, M. A., "Full-wave iterative image reconstruction in photoacoustic tomography with acoustically inhomogeneous media," IEEE T. Med. Imaging 32(6), 10971110 (2013).

[8] Finch, D. and Patch, S. K., "Determining a function from its mean values over a family of spheres," SIAM J. Math. Anal. 35(5), 1213-1240 (2004).

[9] Moczo, P., Kristek, J., Vavryčuk, V., Archuleta, R. J., and Halada, L., "3D heterogeneous staggered-grid finite-difference modeling of seismic motion with volume harmonic and arithmetic averaging of elastic moduli and densities," B. Seismol. Soc. Am. 92(8), 3042-3066 (2002).

[10] Michéa, D. and Komatitsch, D., "Accelerating a three-dimensional finite-difference wave propagation code using GPU graphics cards," Geophys. J. Int. 182(1), 389-402 (2010).

[11] Moczo, P., Kristek, J., Galis, M., Pazak, P., and Balazovjech, M., "The finite-difference and finite-element modeling of seismic wave propagation and earthquake motion," Acta Phys. Slovaca 57(2), 177-406 (2007). 
[12] Komatitsch, D. and Martin, R., "An unsplit convolutional perfectly matched layer improved at grazing incidence for the seismic wave equation," Geophysics 72(5), SM155-SM167 (2007).

[13] Pinton, G., Aubry, J. F., Bossy, E., Muller, M., Pernot, M., and Tanter, M., "Attenuation, scattering, and absorption of ultrasound in the skull bone," Med. Phys. 39(1), 299-307 (2012).

[14] Xu, M. and Wang, L. V., "Universal back-projection algorithm for photoacoustic computed tomography," Phys. Rev. E 71(1), 016706 (2005).

[15] Micikevicius, P., "3D finite difference computation on GPUs using CUDA," in [Proc. GPGPU-2], 79-84, ACM (2009).

[16] Schoonover, R. W., Wang, L. V., and Anastasio, M. A., "Numerical investigation of the effects of shear waves in transcranial photoacoustic tomography with a planar geometry," J. Biomed. Opt. 17(6), 061215106121511 (2012). 